PART II. State of the Field: Advances in Neuroimaging from the 2016 Alzheimer's Imaging Consortium

\title{
Cognitive complaints in older adults at risk for Alzheimer's disease are associated with altered resting-state networks
}

\author{
Joey A. Contreras ${ }^{\mathrm{a}, \mathrm{b}, \mathrm{c}, 1}$, Joaquín Goñi ${ }^{\mathrm{a}}$,b,de,, , Shannon L. Risacher ${ }^{\mathrm{a}, \mathrm{b}}$, Enrico Amico ${ }^{\mathrm{f}}$, \\ Karmen Yoder ${ }^{\mathrm{a}}$, Mario Dzemidzic ${ }^{\mathrm{a}, \mathrm{g}}$, John D. West ${ }^{\mathrm{a}, \mathrm{b}}$, Brenna C. McDonald ${ }^{\mathrm{a}, \mathrm{b}, \mathrm{g}}$, \\ Martin R. Farlow ${ }^{\mathrm{b}, \mathrm{g}}$, Olaf Sporns ${ }^{\mathrm{a}, \mathrm{b}, \mathrm{c}, \mathrm{h}}$, Andrew J. Saykin ${ }^{\mathrm{a}, \mathrm{b}, \mathrm{c}, \mathrm{g}, *}$ \\ ${ }^{a}$ Department of Radiology and Imaging Sciences, Indiana University School of Medicine, Indianapolis, IN, USA \\ ${ }^{b}$ Indiana University Alzheimer Disease Center, Indiana University School of Medicine, Indianapolis, IN, USA \\ ${ }^{c}$ Indiana University Network Science Institute, Indiana University, Bloomington, IN, USA \\ ${ }^{d}$ School of Industrial Engineering, Purdue University, West-Lafayette, IN, USA \\ ${ }^{e}$ Weldon School of Biomedical Engineering, Purdue University, West-Lafayette, IN, USA \\ ${ }^{f}$ Department of Data Analysis, Ghent University, Ghent, Belgium \\ ${ }^{g}$ Department of Neurology, Indiana University School of Medicine, Indianapolis, IN, USA \\ ${ }^{h}$ Department of Psychological and Brain Sciences, Indiana University, Bloomington, IN, USA
}

Abstract

Introduction: Pathophysiological changes that accompany early clinical symptoms in prodromal Alzheimer's disease (AD) may have a disruptive influence on brain networks. We investigated resting-state functional magnetic resonance imaging (rsfMRI), combined with brain connectomics, to assess changes in whole-brain functional connectivity (FC) in relation to neurocognitive variables. Methods: Participants included 58 older adults who underwent rsfMRI. Individual FC matrices were computed based on a 278-region parcellation. FastICA decomposition was performed on a matrix combining all subjects' FC. Each FC pattern was then used as a response in a multilinear regression model including neurocognitive variables associated with AD (cognitive complaint index [CCI] scores from self and informant, an episodic memory score, and an executive function score).

Results: Three connectivity independent component analysis (connICA) components (RSN, VIS, and FP-DMN FC patterns) associated with neurocognitive variables were identified based on prespecified criteria. RSN-pattern, characterized by increased FC within all resting-state networks, was negatively associated with self CCI. VIS-pattern, characterized by an increase in visual resting-state network, was negatively associated with CCI self or informant scores. FP-DMNpattern, characterized by an increased interaction of frontoparietal and default mode networks $(\mathrm{DMN})$, was positively associated with verbal episodic memory.

Discussion: Specific patterns of FC were differently associated with neurocognitive variables thought to change early in the course of $\mathrm{AD}$. An integrative connectomics approach relating cognition to changes in FC may help identify preclinical and early prodromal stages of AD and help elucidate the complex relationship between subjective and objective indices of cognitive decline and differences in brain functional organization.

(C) 2016 The Authors. Published by Elsevier Inc. on behalf of the Alzheimer's Association. This is an open access article under the CC BY-NC-ND license (http://creativecommons.org/licenses/by-nc-nd/ 4.0/).

Keywords: $\quad$ Connectome; Functional connectivity; MRI; Memory; Subjective cognitive decline; Mild cognitive impairment; Alzheimer's disease

\footnotetext{
${ }^{1}$ Authors contributed equally.

*Corresponding author.

E-mail address: asaykin@iupui.edu
} 


\section{Introduction}

Alzheimer's disease (AD) is often recognized as a disconnection disorder where plaques, neurofibrillary tangles, and neurodegeneration of the brain lead to reduced communication and coordination among regions important for cognition [1]. Therefore, brain connectomics studies designed to examine disruptions of connectivity in $\mathrm{AD}$ have become increasingly common [2-7]. Brain connectomics is a field of study that uses graph theory to analyze the ensemble of brain connectivity networks, including functional networks. While neurodegenerative diseases are often studied in a focal manner (i.e., a particular region of interest [ROI] or relatively small neural circuit), brain connectomics provides a more complete systematic view by modeling the entire human brain as a set of networks and assessing whole-brain organizational changes or disruptions.

Functional connectivity (FC) estimates the level of functional coupling of regional brain activity, measured as time series [8], among brain regions regardless of distance or structural connections [9]. This method uses resting-state functional magnetic resonance imaging (rsfMRI) data to measure correlations in low-frequency blood oxygenation leveldependent (BOLD) signal fluctuations between distinct brain regions. Implementation of this technique combined with a gray-matter parcellation allows for extraction of wholebrain FC data. Brain regions can then be further organized into resting-state networks (RSNs), which have been shown to correlate with well-described brain functions and are defined by coordinated neural activity between distinct, spatially separate brain regions [10-13]. Grouping brain regions according to RSN allows for dimension reduction and provides psychophysiologically relevant results [14]. There is evidence that abnormalities in brain networks appear in early stages of $\mathrm{AD}$ [15-17]. Because current evidence indicates that neurodegenerative changes begin before clinical manifestations become apparent [18], it is crucial to identify the earliest changes during preclinical and prodromal stages of disease. Investigating early stages of AD from a FC connectomics perspective may give rise to new hypotheses about underlying pathophysiology that cannot be ascertained using data from isolated regions or circuits $[7,19]$.

Therefore, this study examined FC differences across the continuous spectrum of early-stage $\mathrm{AD}$, from at-risk and prodromal stages to clinical AD dementia with significant cognitive and functional impairment. Participants included those categorized as experiencing subjective cognitive decline (SCD) [20], as well as mild cognitively impaired (MCI) individuals and those diagnosed with AD. SCD participants reported a significant burden of subjective decline in memory and cognition in the absence of psychometric deficits [20,21]. As individuals age, there are increased reports of self-perceived memory decline compared with earlier periods in life [22,23]. However, older adults with SCD have a greater number of complaints and may even subjectively consider their own cognition to be impaired relative to their peers despite objective cognitive test performance (i.e., SCD individuals are only clinically distinguishable from healthy participants in the number of reported cognitive complaints). Relatively high levels of these memory complaints or concerns have been regarded as relevant to the diagnosis of prodromal $\mathrm{AD}$ and are believed to be predictive of subsequent development of amnestic MCI and, at later stages, AD. Therefore, early detection of network changes associated with cognitive complaints could help inform diagnosis and treatment planning and may be useful as a biomarker for enrichment in or as an end point for therapeutic trials.

Because neurodegeneration typically progresses gradually in $\mathrm{AD}$, with subtle transitions between preclinical and prodromal stages rather than discrete changes [24-26], we applied a recently developed analytic framework to identify FC network patterns across early stages of AD. This data-driven methodology, denominated connectivity independent component analysis (connICA) [27] and detailed in the methods section, is distinct from voxel-level and a priori region-based independent component analyses (ICAs) [28]. Our approach can uncover inherent and independent FC patterns that represent different functional RSN features within the population (our group cohort). In addition, it includes an ability to test associations of these FC patterns with subjective and psychometric cognitive parameters relevant to $\mathrm{AD}$. The latter is particularly useful when investigating a continuum of states in which stratification into diagnostic groups may not be clear-cut because of the inherent complexity of diagnostic criteria.

\section{Methods}

\subsection{Participants}

The Indiana University Institutional Review Board approved the study, and written informed consent was obtained from all participants. Participants were older adults from a larger cohort recruited for a longitudinal study of brain aging and memory (Indiana Memory and Aging Study) and were included based on the availability of resting-state imaging data during their first visit. The present sample included 16 participants with significant cognitive complaints despite cognitive test performance within the normal range (SCD group) [21], 21 participants with amnestic MCI, 8 AD patients, and 13 cognitively normal $(\mathrm{CN})$ controls with minimal cognitive complaints. Further details regarding participant recruitment, selection criteria, and characterization are described in previous reports [21,29] and in Table 1. No differences were found between groups regarding age and education (one-way analysis of variance [ANOVA]) or gender (chi-squared test).

\subsection{Neurocognitive variables of interest}

All participants underwent a comprehensive clinical assessment and neuropsychological battery. Five cognitive variables were included in the present analysis: (1) the California Verbal Learning Test (CVLT-II) long-delay free 
Table 1

Demographics

\begin{tabular}{|c|c|c|c|c|c|}
\hline & $\mathrm{CN}$ & SCD & MCI & $\mathrm{AD}$ & $P$-value \\
\hline $\mathrm{N}$ & 13 & 16 & 21 & 8 & - \\
\hline Mean age $(y)$ & $67.15(5.50)$ & $73.38(7.95)$ & $73.33(8.98)$ & $76.38(8.98)$ & NS* \\
\hline Gender $(\mathrm{M} / \mathrm{F})$ & $1 / 12$ & $8 / 8$ & $9 / 13$ & $2 / 6$ & $\mathrm{NS}^{\dagger}$ \\
\hline Mean education (y) & $17.32(1.93)$ & $17.38(1.9)$ & $16(2.8)$ & $16.13(3.7)$ & $\mathrm{NS}^{*}$ \\
\hline Mean CVLT-delayed (episodic memory) & $44.21(7.66)$ & $43.59(7.41)$ & $29.56(8.1)$ & $9.25(11.75)$ & $<.05^{*}$ \\
\hline $\begin{array}{l}\text { Mean WCST_ \# of categories (executive } \\
\text { function) }\end{array}$ & $4.1(1.19)$ & $3.5(1.41)$ & $2.67(1.49)$ & $1.17(0.41)$ & $<.05^{*}$ \\
\hline Mean subject CCI score & $9.97(4.54)$ & $27.92(16.01)$ & $20.85(11.76)$ & $51.29(28.38)$ & $<.05^{*}$ \\
\hline Mean informant CCI score & $7.7(11.46)$ & $15.82(13.41)$ & $14.54(16.1)$ & N/A & $<.05^{*}$ \\
\hline
\end{tabular}

Abbreviations: CN, cognitively normal; SCD, subjective cognitive decline; MCI, mild cognitive impairment; AD, Alzheimer's disease; NS, not significant; CVLT, California Verbal Learning Test; WCST, Wisconsin Card Sorting Test; CCI, cognitive complaint index; N/A, not applicable.

NOTE. The participants of this study were selected from a larger Indiana Memory and Aging Study cohort. Above are the listed means and standard deviations within each group for selected demographic and neurocognitive variables. Between-group differences in age, education, and cognitive variables were tested using a one-way analysis of variance, while chi-squared test was used to detect gender differences between groups.

*One-way ANOVA.

${ }^{\dagger}$ Chi-squared.

recall [30], a measure of episodic memory; (2) the Wisconsin Card Sorting Test (WCST) correct number of categories, a measure of executive function [31]; (3 and 4) a cognitive complaint index (CCI) score from both the participant ("self") and an informant calculated as the percentage of all items given that was endorsed as a complaint [21]; and (5) the highest CCI score from either self or informant, labeled CCImax (see Table 2), included to address the diminished self-awareness that may occur in later stages of AD. Significant differences between groups were detected in all cognitive measures in the expected direction using a one-way ANOVA (see Table 1).

\subsection{MRI data acquisition}

All subjects were imaged on a 3T Siemens Tim Trio MRI scanner with a 12-channel head coil array. A detailed anatomical magnetization-prepared rapid gradient echo (MPRAGE) with whole-brain coverage was acquired using a 3D MPRAGE sequence (repetition time $[\mathrm{TR}] /$ echo time $[\mathrm{TE}]=2300 / 2.98$ $\mathrm{ms}, 220$ sagittal slices with slice thickness $=1.2 \mathrm{~mm}, 1 \times 1$ $\times 1.2 \mathrm{~mm}$ voxels) following the Alzheimer's Disease Neuroimaging Initiative imaging protocol [32]. rsfMRI data were collected with instructions given to subjects to think of nothing in particular while remaining still with their eyes closed. Whole-brain functional images were obtained using a Siemens product gradient echo sequence (echo planar imaging [EPI]; scan duration 6 minutes 9 seconds; 161 BOLD contrastsensitive volumes; TR/TE $=2250 \mathrm{~ms} / 29 \mathrm{~ms} ; 2.5 \times$ $2.5 \times 3.5 \mathrm{~mm}^{3}$ voxels; 39 interleaved axial slices with $3.5 \mathrm{~mm}$ thickness and no gap; GRAPPA acceleration factor 2; 3D PACE prospective motion correction [33]).

\subsection{BOLD preprocessing}

rsfMRI preprocessing procedures, including head motion, were adapted from Power et al. [34]. These included slice timing correction, registration to MPRAGE volume (FLIRT six degrees of freedom [DOFs] and boundary-based registration), detrending, band-pass filtering $(0.009-0.08 \mathrm{~Hz})$, and intensity normalization to mode 1000 . In addition to including motion regressors from the realignment and their derivatives, three image quality control measures (framewise displacement, DVARS, and standard deviation) were applied to identify and remove ("scrubbing") outlier BOLD volumes (D referring to temporal derivative of timecourses, VARS referring to RMS variance over voxels, DVARS). EPI volumes with an excessive $(>50 \%)$ fraction of outliers were dropped from subsequent analyses. This resulted in the exclusion of imaging data from seven subjects (one $\mathrm{CN}$, two $\mathrm{SCD}$, and four $\mathrm{MCI}$ ), with the remaining 58 subjects comprising the final sample. The first five signals obtained from principal component analysis from three tissue compartments were regressed out to address confounding effects of physiologic noise and residual head motion. This procedure relies on eroded masks in the whole-brain gray matter, white matter, and cerebrospinal fluid of the third ventricle and includes global signal regression [35].

The T1-weighted MPRAGE volume of each participant was used to extract the brain (FSL "BET") [36] and perform a sequence of transformations (FSL's FLIRT 6 DOFs, FLIRT 12 DOFs, and nonlinear FNIRT) to the Montreal Neurological Institute (MNI) brain template to provide tissue segmentation. The inverse MNI-to-native transformation then enabled functionally derived brain parcellation [37] into 278 cortical and subcortical ROIs to be performed in native rsfMRI space. Correlation coefficients (Pearson $r$ ) between mean BOLD time series of each ROI pair were sorted into a $278 \times 278$ FC matrix for each participant and used to quantify the degree of connectivity between the paired ROIs. Brain regions were then further ordered into seven independently well-defined cortical RSNs [14], with subcortical and cerebellar regions added for a total of nine networks (Supplemental Fig. S1). This effectively results in a $9 \times 9$ 
FC matrix, where the nine RSNs compromise the cells on the diagonal, and the remaining cells termed "off-diagonal."

\section{5. connICA method}

connICA [27] is a data-driven method based on ICA to obtain cohort-level independent "FC patterns" while estimating the presence of each pattern in an individual subject. Here, ICA is applied directly onto the human connectome domain (as opposed to time-series domain at the voxel level). Specifically, ICA input consists of FC profiles of all participants sorted into a single data set matrix with 58 rows (one row per subject) and 38,503 columns corresponding to the upper triangular elements of each subject's FC matrix (see Fig. 1). These independent patterns extracted from the full FC data set then represent "independent connectome subsystems" that characterize the whole sample and comprise FC profiles of all subjects without any a priori group stratification. ICA decomposition into FC pattern matrix was performed with the fastICA algorithm [38], where the number of components was fixed to 15 as guided by voxel-based ICA studies [39]. Owing to a nondeterministic nature of ICA, we performed 100 connICA runs and kept only independent component patterns deemed to be "robust", that is, appearing in at least 75 out of the 100 runs. In particular, "appearance" required a Pearson correlation coefficient above 0.75 (absolute value) with respect to the first run. This method provides two outputs. The first one, "FC pattern," represents an independent pattern of FC similar to the representation of each subject's FC - a square symmetric matrix with brain regions in rows and columns (and therefore referenced as the FC pattern). However, the FC pattern matrix values are in connectivity units ("loadings"), with their range not restricted to the $[-1,1]$ range, unlike the Pearson correlation coefficient values from the individual FC matrices. The second connICA output is a vector of the signed weights of the FC pattern present on each participant, which quantifies the prominence/presence of the trait in each individual FC matrix as illustrated by Fig. 1 .

connICA yielded six FC patterns. To establish the biological relevance of these patterns, we organized brain regions into canonical $9 \times 9$ RSNs as illustrated by Fig. S2 and detailed in Section 2.4. A pattern was considered in further analyses only if the average FC loadings within at least one element included a significant similarity with either one of the canonical RSNs or their interaction (Supplemental Fig. S2, diagonal or off-diagonal elements, respectively). Specifically, the significance testing included the following steps: (1) generating a frequency histogram of FC loadings from the matrices of all six patterns, with each pattern contributing $(278 * 277) / 2$ values (not including the diagonal); (2) setting a threshold value at the 95th percentile of the FC value distribution; (3) calculating the average FC loading for each pattern matrix in the $9 \times 9$ RSN format; and (4) testing a selection criterion that at least one canonically defined RSN cell must have an average FC 


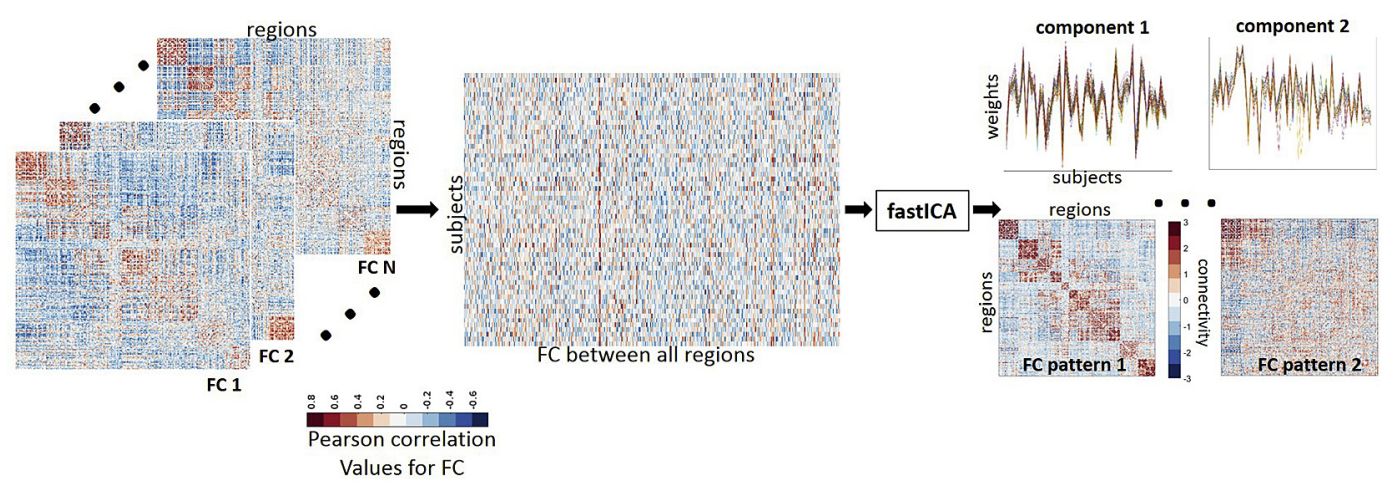

Fig. 1. Connectivity independent component analysis (connICA) methodology. Individual functional connectivity (FC) matrices are concatenated into a group matrix where each row corresponds to one subject and columns are the functional connectivity entries in the FC matrix. FastICA extracts components (i.e., FC patterns) associated to the cohort and their relative weights across subjects. Color bars indicate positive (red) and negative (blue) values; Pearson correlation coefficient values for individual FC matrices (left side of figure) and unit-less connectivity weights for the FC patterns (right side of figure).

value that equals or exceeds the 95th percentile value of 0.72. This significance criterion was met by three out of six FC patterns.

\subsection{Statistical analysis}

The statistical inferences based on multilinear regression analysis were performed using MATLAB [40] function "regress". The subject weights associated with each FC pattern were entered as a response (dependent) variable in an incremental multilinear regression model with up to five predictors (independent variables). The predictors included nuisance variables, including age, gender, and years of education, and a single neurocognitive variable of interest at a time (CVLT long-delay free-recall score, WCST correct number of categories score, CCI-self score, CCI-informant score, and CCImax score). To assess the level of predictability that each neurocognitive variable of interest added, we compared the $\mathrm{R}^{2}$ coefficient value of the multiple regression model run with the neurocognitive variable of interest to the $\mathrm{R}^{2}$ value of the model containing only nuisance variables as predictors.

Finally, the aforementioned cognitive variables that significantly increased the predictability of the chosen FC
A

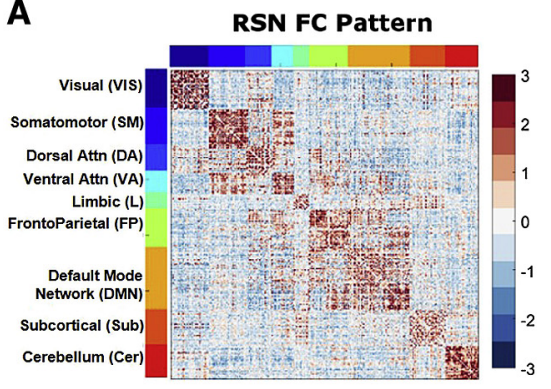

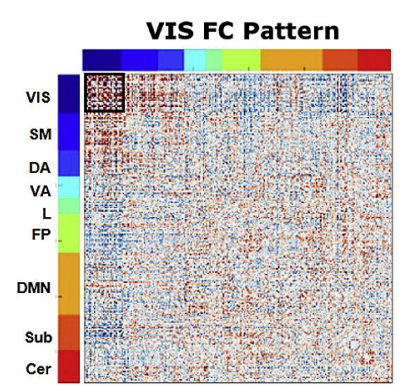

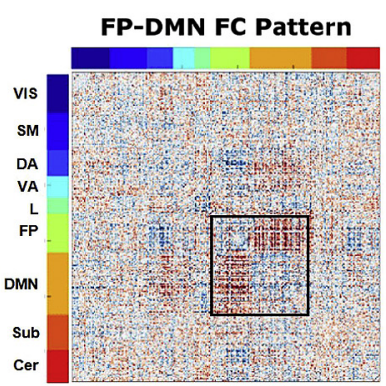

B
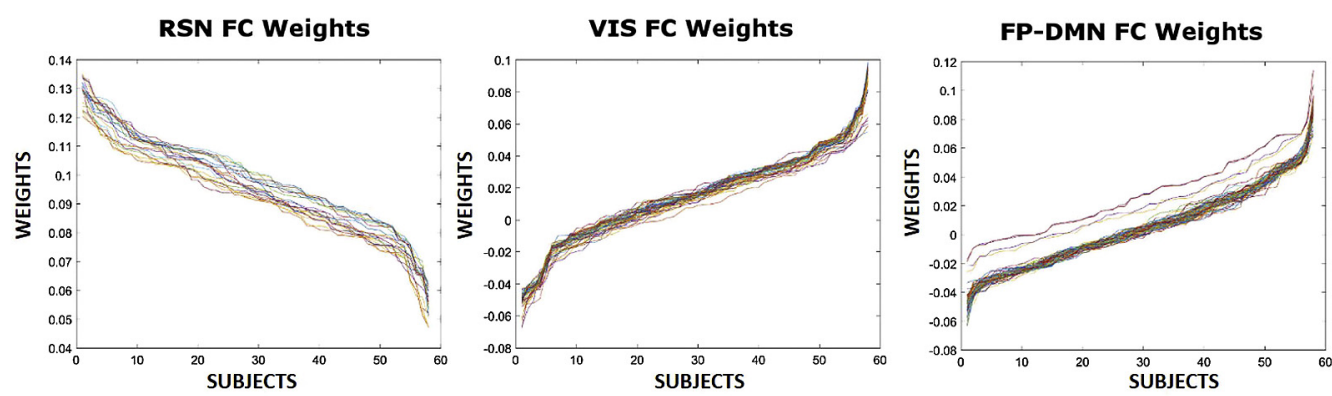

Fig. 2. Robust FC patterns and individual weights as obtained by connICA. (A) Visualization of the FC patterns sorted according to Yeo et al. (2011) functional RSNs. (B) Lines represent the quantified presence of each FC pattern on each individual's functional connectome (across all runs), termed as "weights." All 58 subjects are represented along the $\mathrm{x}$ axis and ordered according to subjects with high presence of the corresponding FC pattern within their functional connectome to those with low presence of the FC pattern within their functional connectome. Additionally, each colored line represents a single ICA run. 
Table 3

Significance values for multilinear regression models

\begin{tabular}{lllll}
\hline Model tested & F-value for model & $P$-value for model & t-Value for predictor & $P$-value for predictor \\
\hline RSN model & 3.40 & $.015^{*}$ & - & -1.69 \\
Age & - & - & 0.29 & .097 \\
Gender & - & - & 0.55 & .773 \\
Education & - & - & -2.39 & .582 \\
CCI-self & - & - & - & $.020^{*}$ \\
VIS model & 1.35 & .267 & 0.44 & - \\
Age & - & - & 0.84 & .659 \\
Gender & - & - & -0.6 & .407 \\
Education & - & - & 2.04 & .550 \\
CCImax & - & - & - & $.047^{*}$ \\
FP-DMN model & 1.96 & .116 & -0.23 &. \\
Age & - & - & 0.63 & .823 \\
Gender & - & - & 2.47 & .535 \\
Education & - & - & 0.27 & $.017^{*}$ \\
CVLT delayed & - & - &
\end{tabular}

NOTE. Table shows significantly predictive multilinear regression models for the three FC patterns.

RSN model : $R S N$ pattern weights $=b_{0}+b_{1} a_{g} e_{1}+b_{2}$ sex $_{2}+b_{3}$ educ $_{3}+b_{4}{\text { cognitive } \text { variable }_{4}}_{4}$

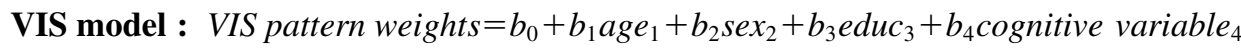

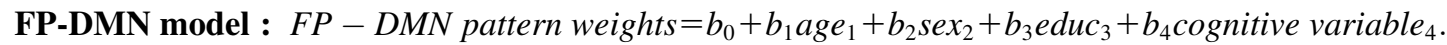

NOTE. Variables included show added significance to individual models. Significance denoted by asterisk $(P<.05)$.

pattern were identified $(P \leq .05)$ to extract FC patterns most associated with cognitive performance and subjective cognitive ratings, which would likely be most sensitive to functional brain changes in preclinical and prodromal $\mathrm{AD}$.

\section{Results}

\subsection{Identification of FC patterns}

The three FC patterns identified using connICA and passing the prespecified selection criteria were (1) resting-state network or RSN-pattern; (2) visual network or VIS-pattern; and (3) frontoparietal and default mode networks or FPDMN pattern. RSN-pattern primarily captures a withinnetwork coherence of the RSNs introduced by Yeo et al [14]. The VIS-pattern was characterized primarily by a significant increase of within-network connectivity in the visual network. Lastly, FP-DMN pattern primarily showed an increased interaction between DMNs and frontoparietal networks (Fig. 2).

\subsection{Relationship of neurocognitive variables of interest and FC patterns}

As expected, the $\mathrm{CN}$ group had the highest CVLTdelayed and WCST performance followed by the SCD group, MCI, and AD groups (Table 1). Similarly, CCI scores differed across diagnoses, in part, by definition as cognitive complaints are part of the criteria for SCD and MCI.

The multilinear regression models including age, gender, education, and neurocognitive variables of interest from Table 2 were performed for each of the three identified FC patterns, with significant results summarized in Table 3.
The first significant finding was observed in the model that included age, gender, education, and CCI-self scores as predictors for subject's weights of the RSN-pattern (Fig. 3A, top panel). The multiple regression model with only nuisance variables as predictors yielded an $R^{2}$ value of 0.11 . However, the inclusion of CCI-self score increased the $R^{2}$ value to 0.20 (Fig. $3 \mathrm{~A}$, middle panel), which was significantly predictive of RSN-pattern weight (Fig. 3A, bottom panel). Specifically, we found that a higher level of selfperceived cognitive decline (e.g., more complaints) was associated with a lower subject weight in the RSN-pattern.

The second significant multilinear regression model included age, gender, education, and CCImax scores as predictors for subjects' weights for the VIS-pattern (Fig. 3B, top panel). If only nuisance variables were used as predictors for the subjects' weights of VIS-pattern, the $\mathrm{R}^{2}$ value was 0.01 . However, the $\mathrm{R}^{2}$ value increased to 0.10 with the addition of CCImax as a predictor, with CCImax significantly predictive within the model for VISpattern weights (Fig. 3B, bottom and middle panels). In this analysis, we observed that a higher maximum complaint score (reported from either the subject or a knowledgeable informant; CCImax) was associated with a lower subject weight score in the VIS-pattern.

The third significant multilinear regression model included age, gender, education, and CVLT scores as predictors for subjects' weights of the FP-DMN-pattern (Fig. 3C, top and bottom panels). Using only nuisance variables as predictors yielded an $\mathrm{R}^{2}$ value of 0.11. CVLT-delayed score increased the $\mathrm{R}^{2}$ value to 0.14 (Fig. 3C, middle panel). In other words, the better the episodic memory performance (i.e., higher CVLT-delayed score), the more that participant 

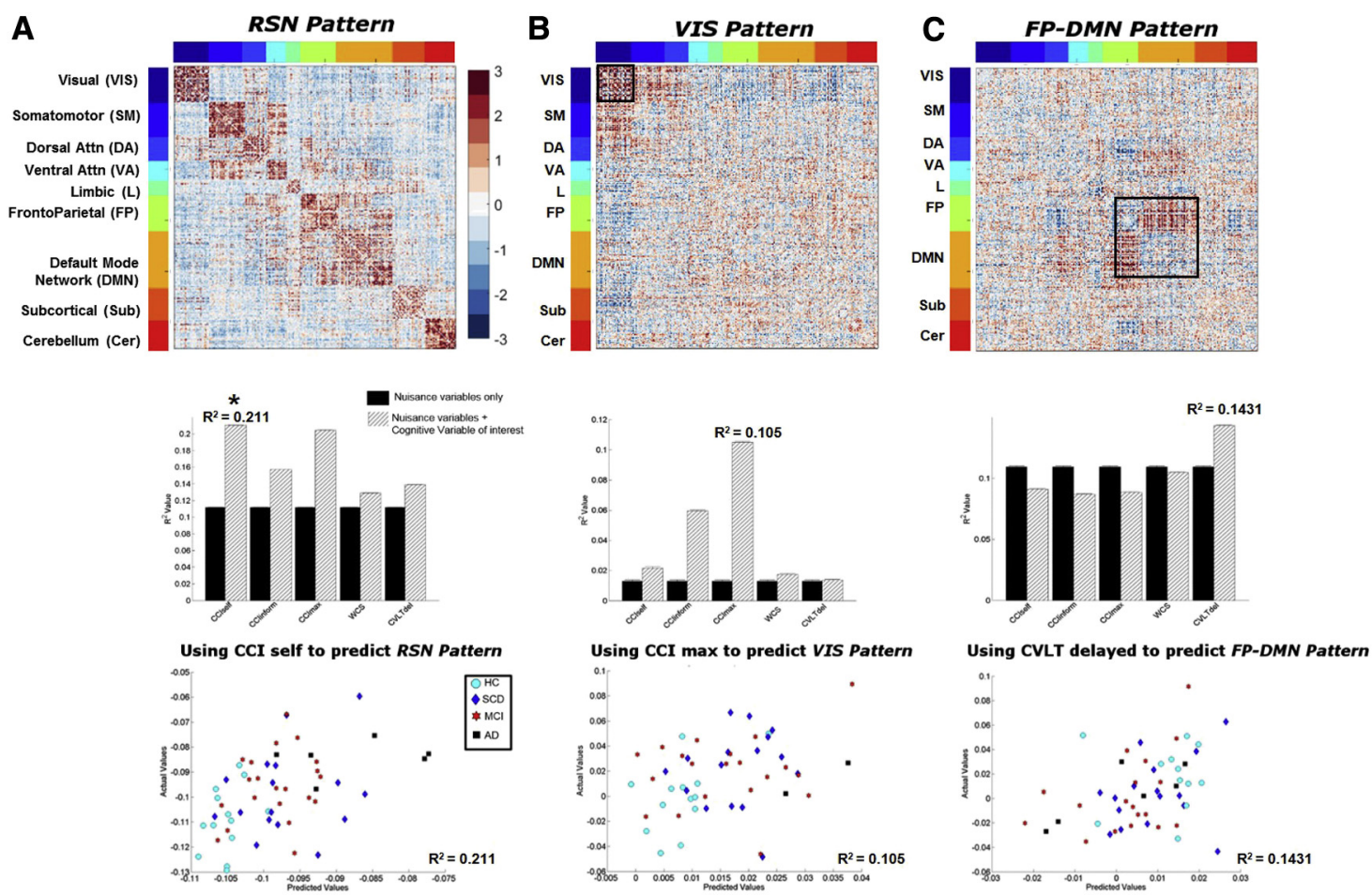

Fig. 3. Relationship of FC patterns and neurocognitive variables of interest. Visualization of the three identified FC patterns (top row). The contributions of neurocognitive variables of interest showing significant increase of the baseline $\mathrm{R}^{2}$ value in the multiple regression models are presented in the bar plots (middle row). A grouped bar plot where black bars indicate the baseline (only age, gender, and education) $\mathrm{R}^{2}$ value, while the hatched patterned bars indicate when an individual neurocognitive variable of interest has been added. The standard error bars were calculated across the 100 ICA runs. $\mathrm{R}^{2}$ value is shown above the cognitive variable that has the greatest increase from the baseline $\mathrm{R}^{2}$ value. The scatter plots (bottom row) show actual versus model-predicted subject weights with different symbols indicating group membership. The multilinear regression models include age, gender, education, and one of the neurocognitive variables of interest. The three columns illustrate the relationships of (A) RSN-pattern and CCI-self score; (B) VIS-pattern and CCImax score, and (C) FP-DMN pattern and CVLT score. These relationships are further detailed in the Results section. Significance denoted by asterisk $(P<.05)$.

exhibited a pattern of increased communication between two important RSNs known to be involved in episodic memory, the DMN and frontoparietal network.

\section{Discussion}

ICA is often used to evaluate the spatiotemporal structure of BOLD data and to extract functional connections from voxels' time series. In a similar vein, we applied a novel, data-driven connICA methodology directly onto functional connectome data sets to model and tease apart common underlying FC patterns of healthy and diseased human brains. We successfully extracted independent FC patterns and quantified the degree to which each pattern was present in each individual's functional connectome. Those individual estimations or pattern weights were used as the response variables in multilinear regression models (one per FC pattern) with nuisance covariates and cognitive performance variables included as predictors. This approach allowed a continuous mapping across different stages of $\mathrm{AD}$ within functional connectomes.

Of the initial six robust independent FC patterns, three (RSN, VIS, and FP-DMN) were considered biologically relevant and retained for further consideration. All three
FC patterns showed associations with neurocognitive status including performance scores. Specifically, we found that SCD measures, considered to be among the earliest indicators of risk for $\mathrm{AD}$, were negatively associated with $\mathrm{FC}$ in several important RSNs. These data indicate the potential utility of connectomics approaches in early detection and diagnosis.

The RSN-pattern, which resembles the canonically defined RSNs [14], was negatively associated with CCIself score after controlling for age, gender, and education. In other words, lower FC within the RSN-pattern was seen in participants with more SCD (i.e., increased complaints). This finding is highly relevant because in early stages of $\mathrm{AD}$, self and informant concerns may precede clinically significant psychometric deficits in cognition, and cognitive complaints are believed to confer an increased risk of cognitive impairment and progression to dementia [20,21]. Our finding suggests that disordered brain function, characterized by decreased coherence in major RSNs, may be related to self-perceived cognitive decline. This result further adds to growing literature that SCD may indeed reflect neuronal changes and suggests that RSN coherence may serve as a good biological predictor of decline $[20,21,41]$. If FC patterns could be used to identify 
individuals in a preclinical state of $\mathrm{AD}$, then this technique has the potential to enhance therapeutic or preventative trials to identify disease-modifying treatments that can stop or slow clinical progression $[42,43]$.

The VIS-pattern had a strong negative association with CCImax score. In other words, higher levels of SCD (taken from either the subject or the informant) are associated with lower within-network FC in the visual network. This result provides evidence that impairment in brain areas responsible for visual cognition may be associated with early indications of cognitive impairment. This is in agreement with studies that have found similar links between visual impairment and early neurodegeneration $[29,44]$. In fact, tests of visual cognition may be particularly sensitive to the effects of $\mathrm{AD}$ [45]. Importantly, we may be able to use the integrity of the FC in the visual network as a diagnostic marker to better characterize or identify early sensory impairments caused by early $\mathrm{AD}$ that may result in decreased quality of life for patients.

Lastly, features of FC in FP-DMN-pattern, which was characterized by strong connectivity of FC between the frontoparietal network and DMN, were positively associated with episodic memory. This result is similar to those from previous studies that suggest that interdependent activity of DMN and parietal regions are involved in memory retrieval $[46,47]$. Thus, our findings and others suggest that strong connections between brain regions in the frontoparietal network and in the DMN are important for better episodic memory performance.

There are limitations to the present study. The sample size is modest, and there is unequal sampling across groups $(\mathrm{CN}$, $\mathrm{SCD}, \mathrm{MCI}$, and AD). Although we did not analyze results based on group, these factors could affect the relative distribution of neurocognitive scores across the sample. Future studies with significantly larger overall and subgroup samples are warranted. Second, we elected to use a specific brain parcellation scheme [37] and referenced our results to a particular set of RSNs [14]. It is possible that alternative anatomic and functional templates would return different results. Thus, future studies including multiple brain parcellation schemes and RSN definitions would help determine the validity of our findings. Third, the ICA approach is still being optimized, in particular with regards to the upper limit of components that the algorithm can extract. Fourth, permutation tests and cross-validation of the clinical cohort would enable nonparametric estimation of significance [48] and provide a data-driven approach to multiple testing correction. Fifth, we were unable to incorporate data from clinically relevant biomarkers such as $\beta$-amyloid and tau. To determine the impact of hallmark AD biomarkers on FC patterns, future studies will incorporate plasma and cerebrospinal fluid concentrations of amyloid and tau, as well as positron emission tomography data with radioligands that bind to these proteins.

Finally, the field of brain connectomics has great promise for elucidating the complex relationships between cognitive decline and FC patterns. This approach has strong potential to generate clinically relevant biomarkers for brain function in prodromal $\mathrm{AD}$ and other neurodegenerative disorders.

\section{Acknowledgments}

This study was supported, in part, by National Institute on Aging grants R01 AG019771, P30 AG10133, and K01 AG049050, and the Alzheimer's Association, Indiana Clinical and Translational Science Institute (UL1 TR001108), and Indiana University-IU Health Strategic Research Initiative and the IU Network Science Institute (IUNI). We thank the staff of the Indiana Memory and Aging Study, the Indiana Alzheimer Disease Center, as well as the imaging staff of the Indiana Institute for Biomedical Imaging Sciences and the IU Center for Neuroimaging. We are very grateful to the study participants and their families who made this research possible.

\section{Supplementary data}

Supplementary data related to this article can be found at http://dx.doi.org/10.1016/j.dadm.2016.12.004.

\section{RESEARCH IN CONTEXT}

1. Systematic review: This study examined functional connectivity (FC) differences across the spectrum of early-stage $\mathrm{AD}$, from at-risk and prodromal stages to clinical $\mathrm{AD}$ dementia with significant cognitive and functional impairment. Because neurodegeneration typically progresses grad- ually in $\mathrm{AD}$, with subtle transitions between preclinical and prodromal stages rather than discrete drops, we applied a recently developed analytic framework to identify FC network patterns across early stages of AD. Specifically, a data-driven methodology, denominated connICA, was used to uncover inherent and independent FC patterns within the population and their associations with subjective and psychometric cognitive parameters relevant to $\mathrm{AD}$.

2. Interpretation: Our finding suggests that disordered brain function, characterized by decreased coherence in major resting-state networks, may be related to self- perceived cognitive decline.

3. Future directions: Future studies with significantly larger overall group and subgroup samples are warranted along with optimization of this novel ICA approach. Finally, FC patterns could be used to identify individuals in a preclinical state of $\mathrm{AD}$, helping to enhance therapeutic or preventative trials to identify disease-modifying treatments that can stop or slow clinical progression. 


\section{References}

[1] Delbeuck X, Van der Linden M, Collette F. Alzheimer's disease as a disconnection syndrome? Neuropsychol Rev 2003;13:79-92.

[2] Contreras JA, Goñi J, Risacher SL, Sporns O, Saykin AJ. The Structural and Functional Connectome and Prediction of Risk for Cognitive Impairment in Older Adults. Curr Behav Neurosci Rep 2015;2:234-45.

[3] Crossley NA, Mechelli A, Scott J, Carletti F, Fox PT, McGuire P, et al. The hubs of the human connectome are generally implicated in the anatomy of brain disorders. Brain 2014;137:2382-95.

[4] Dai Z, Yan C, Li K, Wang Z, Wang J, Cao M, et al. Identifying and Mapping Connectivity Patterns of Brain Network Hubs in Alzheimer's Disease. Cereb Cortex 2015;25:3723-42.

[5] Prescott JW, Guidon A, Doraiswamy PM, Roy Choudhury K, Liu C, Petrella JR. The Alzheimer structural connectome: changes in cortical network topology with increased amyloid plaque burden. Radiology 2014;273:175-84.

[6] Thomas JB, Brier MR, Bateman RJ, Snyder AZ, Benzinger TL, Xiong $\mathrm{C}$, et al. Functional connectivity in autosomal dominant and late-onset Alzheimer disease. JAMA Neurol 2014; 71:1111-22.

[7] Sepulcre J, Johnson KA, Sperling R. Tau and AB deposits relate to distinctive functional connectivity disruptions in the elderly brain. Alzheimers Dement 2014;10:P159-60.

[8] van den Heuvel MP, Hulshoff Pol HE. Exploring the brain network: a review on resting-state fMRI functional connectivity. Eur Neuropsychopharmacol 2010;20:519-34.

[9] Hagmann P, Cammoun L, Gigandet X, Meuli R, Honey CJ, Wedeen VJ, et al. Mapping the structural core of human cerebral cortex. PLoS Biol 2008;6:e159.

[10] Damoiseaux JS, Rombouts SA, Barkhof F, Scheltens P, Stam CJ, Smith SM, et al. Consistent resting-state networks across healthy subjects. Proc Natl Acad Sci U S A 2006;103:13848-53.

[11] Catani M, Thiebaut de Schotten M, Slater D, Dell'Acqua F. Connectomic approaches before the connectome. Neuroimage 2013;80:2-13.

[12] Sporns O. The human connectome: origins and challenges. Neuroimage 2013;80:53-61.

[13] Smith SM, Vidaurre D, Beckmann CF, Glasser MF, Jenkinson M, Miller KL, et al. Functional connectomics from resting-state fMRI. Trends Cogn Sci 2013;17:666-82.

[14] Yeo BT, Krienen FM, Sepulcre J, Sabuncu MR, Lashkari D, Hollinshead M, et al. The organization of the human cerebral cortex estimated by intrinsic functional connectivity. J Neurophysiol 2011; 106:1125-65.

[15] Cai S, Chong T, Peng Y, Shen W, Li J, von Deneen KM, et al. Altered functional brain networks in amnestic mild cognitive impairment: a resting-state fMRI study. Brain Imaging Behav 2016; [Epub ahead of print].

[16] Zhu D, Li K, Terry DP, Puente AN, Wang L, Shen D, et al. Connectomescale assessments of structural and functional connectivity in MCI. Hum Brain Mapp 2014;35:2911-23.

[17] Zhan Y, Ma J, Alexander-Bloch AF, Xu K, Cui Y, Feng Q, et al. Longitudinal Study of Impaired Intra- and Inter-Network Brain Connectivity in Subjects at High Risk for Alzheimer's Disease. J Alzheimers Dis 2016;52:913-27.

[18] Jack CR Jr, Knopman DS, Jagust WJ, Petersen RC, Weiner MW, Aisen PS, et al. Update on hypothetical model of Alzheimer's disease biomarkers. Lancet Neurol 2013;12:207.

[19] Brier MR, Thomas JB, Fagan AM, Hassenstab J, Holtzman DM, Benzinger TL, et al. Functional connectivity and graph theory in preclinical Alzheimer's disease. Neurobiol Aging 2014;35:757-68.

[20] Jessen F, Amariglio RE, Van Boxtel M, Breteler M, Ceccaldi M, Chételat $\mathrm{G}$, et al. A conceptual framework for research on subjective cognitive decline in preclinical Alzheimer's disease. Alzheimers Dement 2014; 10:844-52.
[21] Saykin AJ, Wishart HA, Rabin LA, Santulli RB, Flashman LA, West JD, et al. Older adults with cognitive complaints show brain atrophy similar to that of amnestic MCI. Neurology 2006;67:834-42.

[22] Koppara A, Wagner M, Lange C, Ernst A, Wiese B, Konig HH, et al. Cognitive performance before and after the onset of subjective cognitive decline in old age. Alzheimers Dement (Amst) 2015;1:194-205.

[23] Jonker C, Geerlings MI, Schmand B. Are memory complaints predictive for dementia? A review of clinical and population-based studies. Int J Geriatr Psychiatry 2000;15:983-91.

[24] Dickerson BC, Sperling RA, Hyman BT, Albert MS, Blacker D. Clinical prediction of Alzheimer disease dementia across the spectrum of mild cognitive impairment. Arch Gen Psychiatry 2007;64:1443-50.

[25] Chan M, Tay L, Chong MS. Amnestic mild cognitive impairment and early Alzheimer's disease in an asian memory clinic - evidence for a clinical spectrum. Dement Geriatr Cogn Dis Extra 2011;1:113-23.

[26] Montine TJ, Phelps CH, Beach TG, Bigio EH, Cairns NJ, Dickson DW, et al. National Institute on Aging-Alzheimer's Association guidelines for the neuropathologic assessment of Alzheimer's disease: a practical approach. Acta Neuropathol 2012;123:1-11.

[27] Amico E, Marinazzo D, DiPerri C, Heine L, Annen J, Martial C, et al. Mapping the functional connectome traits of levels of consciousness. NeuroImage 2017;148:201-11.

[28] Calhoun VD, Liu J, Adali T. A review of group ICA for fMRI data and ICA for joint inference of imaging, genetic, and ERP data. Neuroimage 2009;45:S163-72.

[29] Risacher SL, Wudunn D, Pepin SM, MaGee TR, McDonald BC, Flashman LA, et al. Visual contrast sensitivity in Alzheimer's disease, mild cognitive impairment, and older adults with cognitive complaints. Neurobiol Aging 2013;34:1133-44.

[30] Delis DC, Kramer JH, Kaplan E, Thompkins BA. CVLT, California Verbal Learning Test: Adult Version: Manual. Psychological Corporation; 1987.

[31] Berg EA. A simple objective technique for measuring flexibility in thinking. J Gen Psychol 1948;39:15-22.

[32] Jack CR Jr, Bernstein MA, Fox NC, Thompson P, Alexander G, Harvey D, et al. The Alzheimer's Disease Neuroimaging Initiative (ADNI): MRI methods. J Magn Reson Imaging 2008;27:685-91.

[33] Thesen S, Heid O, Mueller E, Schad LR. Prospective acquisition correction for head motion with image-based tracking for real-time fMRI. Magn Reson Med 2000;44:457-65.

[34] Power JD, Mitra A, Laumann TO, Snyder AZ, Schlaggar BL, Petersen SE. Methods to detect, characterize, and remove motion artifact in resting state fMRI. Neuroimage 2014;84:320-41.

[35] Power JD, Barnes KA, Snyder AZ, Schlaggar BL, Petersen SE Spurious but systematic correlations in functional connectivity MRI networks arise from subject motion. Neuroimage 2012; 59:2142-54.

[36] Jenkinson M, Pechaud M, Smith S. BET2: MR-based estimation of brain, skull and scalp surfaces. Eleventh annual meeting of the organization for human brain mapping: Toronto, ON; 2005. p. 167.

[37] Shen X, Tokoglu F, Papademetris X, Constable RT. Groupwise whole-brain parcellation from resting-state fMRI data for network node identification. Neuroimage 2013;82:403-15.

[38] Hyvärinen A. Fast ICA for noisy data using Gaussian moments. Circuits and Systems, 1999 ISCAS'99 Proceedings of the 1999 IEEE International Symposium on: IEEE; 1999. p. 57-61.

[39] Calhoun VD, Adali T, Pearlson GD, Pekar JJ. A method for making group inferences from functional MRI data using independent component analysis. Hum Brain Mapp 2001;14:140-51.

[40] MathWorks Inc. MATLAB: the language of technical computing. Desktop tools and development environment, version 7. MathWorks; 2005.

[41] Reisberg B, Gauthier S. Current evidence for subjective cognitive impairment ( $\mathrm{SCI}$ ) as the pre-mild cognitive impairment (MCI) stage of subsequently manifest Alzheimer's disease. Int Psychogeriatr 2008:20:1-16.

[42] Kivipelto M, Solomon A, Ahtiluoto S, Ngandu T, Lehtisalo J, Antikainen R, et al. The Finnish Geriatric Intervention Study to 
Prevent Cognitive Impairment and Disability (FINGER): study design and progress. Alzheimers Dement 2013;9:657-65.

[43] Richard E, Andrieu S, Solomon A, Mangialasche F, Ahtiluoto S, Moll van Charante EP, et al. Methodological challenges in designing dementia prevention trials - the European Dementia Prevention Initiative (EDPI). J Neurol Sci 2012;322:64-70.

[44] Fujimori M, Imamura T, Yamashita H, Hirono N, Ikejiri Y, Shimomura T, et al. Age at onset and visuocognitive disturbances in Alzheimer disease. Alzheimer Dis Assoc Disord 1998;12:163-6.
[45] Freeman RQ, Giovannetti T, Lamar M, Cloud BS, Stern RA, Kaplan E, et al. Visuoconstructional problems in dementia: contribution of executive systems functions. Neuropsychology 2000;14:415-26.

[46] Buckner RL, Wheeler ME. The cognitive neuroscience og remembering. Nat Rev Neurosci 2001;2:624-34.

[47] Wagner AD, Shannon BJ, Kahn I, Buckner RL. Parietal lobe contributions to episodic memory retrieval. Trends Cogn Sci 2005;9:445-53.

[48] Fornito A, Zalesky A, Bullmore E. Fundamentals of Brain Network Analysis. London: Academic Press; 2016. 